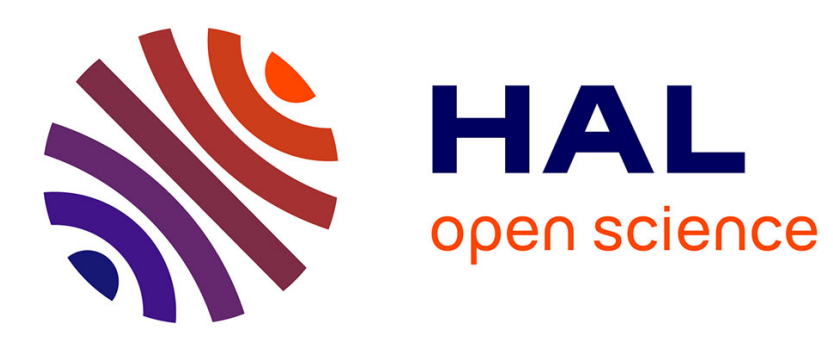

\title{
Colloidal dispersions of $\alpha$-crystallin proteins. - I. Small angle X-ray analysis of the dispersion structure
}

\author{
A. Tardieu, D. Laporte, M. Delaye
}

\section{To cite this version:}

A. Tardieu, D. Laporte, M. Delaye. Colloidal dispersions of $\alpha$-crystallin proteins. - I. Small angle X-ray analysis of the dispersion structure. Journal de Physique, 1987, 48 (7), pp.1207-1215. 10.1051/jphys:019870048070120700 . jpa-00210545

\section{HAL Id: jpa-00210545 https://hal.science/jpa-00210545}

Submitted on 1 Jan 1987

HAL is a multi-disciplinary open access archive for the deposit and dissemination of scientific research documents, whether they are published or not. The documents may come from teaching and research institutions in France or abroad, or from public or private research centers.
L'archive ouverte pluridisciplinaire HAL, est destinée au dépôt et à la diffusion de documents scientifiques de niveau recherche, publiés ou non, émanant des établissements d'enseignement et de recherche français ou étrangers, des laboratoires publics ou privés. 
Classification

Physics Abstracts

$82.70-36.20$

\title{
Colloidal dispersions of $\alpha$-crystallin proteins. I. Small angle X-ray analysis of the dispersion structure
}

\author{
A. Tardieu ( $\left(^{a}\right)$, D. Laporte $\left({ }^{b}\right)$ and M. Delaye $\left({ }^{c}\right)$ \\ ( $\left.{ }^{a}\right)$ Centre de Génétique Moléculaire, C.N.R.S., 91190 Gif sur Yvette, France \\ (b) Laboratoire L. Brillouin, C.E.N. Saclay, 91190 Gif sur Yvette, France \\ (c) Laboratoire de Physique des Solides, Bât. 510, Université Paris-Sud, 91405 Orsay, France
}

(Reçu le 2 février 1987, accepté le 12 mars 1987)

\begin{abstract}
Résumé. - Nous avons étudié, par diffusion des rayons $\mathrm{X}$, la structure des dispersions colloïdales de protéines spécifiques du cristallin de l'œil, les $\alpha$-cristallines, à deux forces ioniques différentes et pour des concentrations allant jusqu'à $0,20 \mathrm{~g} / \mathrm{cm}^{3}$. Le modèle de RMSA (renormalized mean spherical approximation), couplé à un potentiel du type Verwey et Overbeek, rend compte correctement des données expérimentales dans une large gamme de vecteurs d'ondes. L'évaluation du volume exclu et de la charge des macromolécules obtenue à partir du modèle est en bon accord avec les données déjà publiées. Nous discutons les conditions d'application du modèle au cas des $\alpha$-cristallines. L'ensemble des résultats obtenus montre que les $\alpha$-cristallines et plus généralement sans doute les protéines, constituent une source naturelle de systèmes modèles pour l'étude des dispersions colloïdales.
\end{abstract}

\begin{abstract}
The structure of colloidal dispersions of eye lens $\alpha$-crystallin proteins was investigated using small angle X-ray scattering. The renormalized mean spherical approximation (RMSA), combined with a VerweyOverbeek (VO) potential, was used to analyse the results obtained at two different ionic strengths, for concentrations up to $0.20 \mathrm{~g} / \mathrm{cm}^{3}$. This model correctly accounts for the experimental data over a large scattering domain. It provides as best fit parameters an evaluation of the excluded volume and of the charge of the macromolecules which compares well with the values found in the literature. The adequacy of the model for the description of the interactions between $\alpha$-crystallin particles is discussed. The whole of the results obtained indicate that $\alpha$-crystallins, and possibly proteins in general, provide a natural.source of model systems to study colloidal dispersions.
\end{abstract}

\section{Introduction.}

Structure of aqueous dispersions of colloidal particles (such as charged micelles or microemulsions) is often analysed by scattering techniques. In contrast, this type of analysis is only rarely applied to protein solutions. The reason is probably that in biology the structure-function relationship is most often sought in local structural properties of the protein particle itself, which are more conveniently studied in «ideal» solutions or in crystals. Some biological functions, however, are linked to macroscopic properties of the cellular medium and the solution structure then becomes the relevant information. This was shown to be true for eye lens transparency, which is determined by the spatial organisation of the lens proteins in the cytoplasm [1].

$\alpha$-crystallins, which are the colloidal particles considered in the present work, are the largest and most abundant species of eye lens specific proteins and represent as such a key-factor in eye lens transparency. $\alpha$-crystallins are negatively charged polydisperse oligomers of a 20000 dalton molecular weight subunit. Previous studies led to a quaternary structure model in which the subunits are organized in 3 concentric spherical shells [2]. $\alpha$-crystallins can thus be crudely described as quasi-spherical particles, with an outer diameter of the order of 180 to $200 \AA$. In the preparation used for the present investigation, $\alpha$-crystallins had, on average, 55 subunits and a size polydispersity of about $10 \%$.

We used small angle $\mathrm{X}$-ray scattering to measure the structure factors of $\alpha$-crystallin solutions as a function of concentration, at two ionic strengths (I). The experiments were analysed within the 
framework of the renormalized mean spherical approximation (RMSA), with the Verwey-Overbeek (VO) potential to describe screened coulombic interactions as developed by Hayter and Penfold [3] and modified by Hansen and Hayter [4]. In this article, some representative data were chosen to discuss whether such a model is suited to analysis of $\alpha$-crystallin solutions. It turned out that this was indeed the case. $\alpha$-crystallins thus represent a good model system for colloidal dispersions. A second article is devoted to the dynamics of these $\alpha$-crystallin dispersions [5].

\section{Experimental procedures.}

2.1 PREPARATION OF $\alpha$-CRYSTALLIN DISPERSIONS. - $\alpha$-crystallins were extracted from the cortex (i.e. periphery) of fresh calf lenses (obtained from the Blois slaughterhouse). The cortex was first homogenized in a physiological buffer B1 (B1 = phosphate buffer pH 6.8, ionic strength $I=150$ mequiv, supplemented with $\mathrm{NaN}_{3}$ to prevent bacterial growth, DTT $\left({ }^{1}\right)$ to prevent oxydation and PMSF $\left({ }^{1}\right)$ to prevent enzymatic degradation). Cortex solutions were then centrifuged $30 \mathrm{~min}$ at $10000 \mathrm{~g}$ to clear out membrane fragments, applied to a fractogel (TSK HW $55 \mathrm{~S})$ column $(\varphi=2.6 \mathrm{~cm}, l=100 \mathrm{~cm})$ and eluted with B1 buffer. Under these conditions, $\alpha$-crystallins elute as a first broad peak [2]. Top fractions of the peak were pooled for further experiments resulting in a population of macromolecules with about $30 \%$ mass polydispersity, i.e. about $10 \%$ size polydispersity.

$\alpha$-crystallin solutions were first concentrated using an Amicon ultrafiltration cell with a YM 100 membrane and then by ultracentrifugation (180000 g, $\left.4 \mathrm{~h}, 20^{\circ} \mathrm{C}\right)$; the resulting pellet was a viscous dispersion of $\alpha$-crystallins $\left(c=0.35 \mathrm{~g} / \mathrm{cm}^{3}\right)$. This dispersion was divided into two parts. The first one was diluted with B1 buffer to reach various concentrations. The second one was first dialyzed for 2 days against a low ionic strength buffer B2 (10 mM phosphate, pH 6.8, I = 17 mequiv). Lower concentrations were then obtained in this ionic strength series by dilution with B2. The concentration of each dispersion was deduced from refractive index measurement using the relations :

$$
\begin{aligned}
& n=1.3342+0.183 c \text { for } I=150 \text { mequiv } \\
& n=1.3328+0.186 c \text { for } I=17 \text { mequiv }
\end{aligned}
$$

where $c$ is given in $\mathrm{g} / \mathrm{cm}^{3}$.

The 3 concentrations used in the present study were, for each ionic strength, of the order of 0.01 , 0.10 and $0.20 \mathrm{~g} / \mathrm{cm}^{3}$.

$\left({ }^{1}\right)$ DTT $=$ dithiothreitol, PMSF $=$ phenylmethansulfonylfluorid.
2.2 SMALl ANGLE X-RAY SCATTERING. - Experiments were performed using the small angle camera D24 of the Synchrotron Radiation Laboratory Lure (Orsay). The X-ray beam was monochromated ( $\lambda=1.608 \AA$, K-edge of Co) and focussed with a bent germanium crystal. Point collimation geometry was used. The X-ray beam had a cross-section of $0.5 \mathrm{~mm} \times 1.0 \mathrm{~mm}$ at the detector level. The detector was a linear position sensitive detector with delay line readout linked to a data-acquisition system designed at the E.M.B.L. [6] (Heidelberg and Hamburg). A $1 \mathrm{~mm}$ slit was placed in front of the detector to maintain point collimation conditions.

Sample to detector distance was $1 \mathrm{~m}$, ds/channel was $1.355 \times 10^{-4} \AA^{-1}(s=2 \sin \theta / \lambda$, with $2 \theta$ the scattering angle) and the recorded $s$ range was $1.3 \times 10^{-3}<s<2.2 \times 10^{-2} \AA^{-1}$ (the corresponding range for $q=2 \pi s$ is $8.2 \times 10^{5}<q<1.4 \times$ $10^{7} \mathrm{~cm}^{-1}$ ).

Samples were contained in calibrated quartz capillary tubes, about $1 \mathrm{~mm}$ in diameter. Experiments were performed at room temperature. Average exposure time for samples with $c>0.10 \mathrm{~g} / \mathrm{cm}^{3}$ was from 10 to $20 \mathrm{~min}$. The $c=0.01 \mathrm{~g} / \mathrm{cm}^{3}$ samples and the background were recorded several times during the run, for a total exposure time of $100 \mathrm{~min}$.

After background subtraction, the experimental intensities $I_{\exp }(c, s)$ were put on an absolute scale, $i_{\mathrm{n}}(c, s) / c_{\mathrm{e}}$, which corresponds to normalization to one electron of solute particle ( $c_{\mathrm{e}}$ is the concentration in electrons of solute/electrons of solution). For the sake of simplicity, these normalized intensities will be referred to in the following as $I(c, s)$. As detailed in reference [7], this absolute scale is easily calculated from the experimental parameters :

$$
I(c, s)=i_{\mathrm{n}}(c, s) / c_{\mathrm{e}}=I_{\exp }(c, s) / K E_{0} \varphi_{\mathrm{c}} c
$$

where $K$ takes into account physical constants, $E_{0}$ is the energy of the incident beam, measured by reference to a previously calibrated carbon black and $\varphi_{c}$ is the thickness of the capillary tube, determined by measurement of water absorption.

After absolute scale normalization, $s^{4} I(c, s)$ was plotted as a function of $s^{4}$. As expected for proteins, linear behaviour $\left(s^{4} I(s)=A+B s^{4}\right)$ was found for $s>0.012 \AA^{-1}$, allowing us to determine $A$ and $B$ for each curve [8]. The $A$ term measures the solutesolvent interface. The $B$ term takes into account residual electron density fluctuations as well as possible uncertainty on the coefficient of the background to be subtracted (note that the background is flat for large $s$ values). The $B$ term was subtracted from each curve, after which the normalized scattering curves recorded at different concentrations and plotted on a log scale were all parallel for $s>0.0095 \AA^{-1}$.

The coefficient needed to render the scattering curves strictly identical in this $s$ region did not show 
any systematic variation with concentration and remained equal to 1 within $10 \%$. The need to introduce this coefficient was ascribed to uncertainties in the absolute scale determination and the scattering curves were renormalized to the same integrated intensity for $s>0.0095 \AA^{-1}$.

With the normalization used, the intensity scattered by a monodisperse solution of spherical particles, whose size and shape are invariant with concentration, is expected to vary as a function of concentration according to :

$$
I(c, s)=I(0, s) \times S(c, s)
$$

where $I(0, s)$ is the form factor of the particle and $S(c, s)$ the structure factor of the solution.

Near the origin of $s$, the form factor of globular particles may be written :

$$
I(0, s)=m\left(1-\rho_{0} \psi\right)^{2}\left(1-\frac{4}{3} \pi^{2} R_{\mathrm{g}}^{2} s^{2}+\cdots\right)
$$

where $m$ is the number of electrons of one particle, $R_{\mathrm{g}}$ the radius of gyration, $\rho_{0}$ is the electron density of the solvent and $\psi$ the partial electronic volume of the particle.

The experimental form factor is usually obtained as the scattering curve extrapolated to zero concentration. In the present case, we took as the form factor, in the range $s>1 / 2 \pi R_{\mathrm{g}}=2.4 \times 10^{-3} \AA^{-1}$, the normalized scattering curve obtained at $c=$ $0.01 \mathrm{~g} / \mathrm{cm}^{3}$ and $I=150$ mequiv. Average molecular weight $M$ and radius of gyration were determined from the Guinier plot of this curve drawn from $s=1 / 2 \pi R_{\mathrm{g}}=2.4 \times 10^{-3} \AA^{-1}$ and $s=2 / 2 \pi R_{\mathrm{g}}=$ $4.8 \times 10^{-3} \AA^{-1}$ using $\psi=2.3 \mathrm{e} \AA^{-3}$ and $M / m=$ 1.87 (typical values for proteins). Previous studies performed as a function of concentration for $0.003<c<0.01 \mathrm{~g} / \mathrm{cm}^{3}$ [2] had shown that the Guinier plot was linear in this $s$-range and independent of $c$. The Guinier plot was used to extrapolate the experimental form factor at lower $s$ values. Experimental structure factors $S_{\text {exp }}(c, s)$ were determined according to equation (2), using the experimental form factor as $I(0, s)$.

2.3 MODELLING THE STRUCTURE FACTORS WITH RMSA AND VO POTENTIAL. - RMSA combined with a VO potential has been shown adequate to describe the structure of macroion solutions in which the size of the macroion is large enough to dominate many of the solution properties and its charge large enough to make Van der Waals attraction negligible [4]. This model also neglects departure from sphericity and polydispersity. The validity of these assumptions for our system are dealt with in the discussion.

The screened Coulomb potential is described by :

$$
\begin{array}{r}
v(r)=\left(\pi \varepsilon_{0} \varepsilon \sigma^{2} \psi_{0}^{2} / r\right) \exp [-k(r-\sigma)] \\
\text { for } r>\sigma
\end{array}
$$

where

$\sigma$ is the hard sphere diameter of the colloidal particle

$r$ the interparticle center to center distance

$\varepsilon$ the dielectric constant of the solvent medium

$k$ is the Debye-Hückel inverse screening length :

$$
k=1 / \lambda_{\mathrm{D}}=\left[2 e^{2} 10^{3} \mathcal{N} I / \varepsilon_{0} \varepsilon k_{\mathrm{B}} T\right]^{1 / 2}
$$

with $e$ the electronic charge, $\mathcal{N}$ the Avogadro number, $k_{\mathrm{B}}$ the Boltzmann constant and $T$ the absolute temperature, $I=1 / 2 \sum c_{i} Z_{i}^{2}$ the ionic strength of the solvent ( $I$ in equiv; we neglect possible variations of $I$ with macroion concentration c).

$c_{i}$ and $Z_{i}$ the molar concentration $\left(\mathrm{mol} . \mathrm{1}^{-1}\right)$ and the valence of the $i$-th ion. Typically in an aqueous solution at $25^{\circ} \mathrm{C}$, one finds

$$
\lambda_{\mathrm{D}}(\AA)=3 / \sqrt{I \text { (equiv) }}
$$

$\psi_{0}$ is the surface potential related, in the DebyeHückel approximation, to an «effective» particle charge $Z e$ through

$$
\psi_{0}=Z e / \pi \varepsilon_{0} \varepsilon \sigma(2+k \sigma) .
$$

Typically for aqueous solutions one has, $\sigma$ being expressed in $\AA$

$$
\psi_{0}(\mathrm{mV})=720 Z / \sigma(2+k \sigma) .
$$

Theoretical structure factors $S_{\text {th }}(c, s)$ were calculated using the fortran package developed by Hayter and Penfold [3] and modified by Hansen and Hayter [4], implanted on an IBM computer at the Centre Inter-Régional de Calcul Electronique (CIRCE, Orsay). The fitting parameters were adjusted numerically, one parameter at a time as explained in Results, by minimizing $\sum\left|S_{\text {th }}-S_{\text {exp }}\right|$ in the desired $s$ region.

This RMSA model and the potential it uses involves several approximations. First, RMSA uses an approximate integral equation with a pair potential $v(r)$ which gives inexact results at very high concentrations. Also, RMSA is a «one-component model » which neglects the molecular structure of the solvent. As shown by references [9] and [10], finite size counter ions do screen the macromolecular charge slightly less efficiently than point size ions, with the result of making the interaction peak of the structure factor slightly higher than in the point size approximation. However, the $\alpha$-crystallin radius being some 20 times larger than counterion radii, such an effect remains negligible.

The calculation of the VO potential is derived from a «superposition principle », which is only valid when the diffuse layers do not significantly 
overlap, i.e. when the average centre-to-centre distance $d$ satisfies :

$$
d-\sigma>2 \lambda_{\mathrm{D}}=2 / k
$$

Calculating the number $n$ of particles per unit volume as $6 \phi / \pi \sigma^{3}$ and taking $d$ as $n^{-1 / 3}$, the condition (7) is fulfilled up to $\phi=0.45$ at $I=150$ mequiv and $\phi=0.3$ at $I=17$ mequiv ( $\phi$ is the excluded volume fraction).

Finally, the pertinence of the VO potential [11] for describing the repulsive interactions between $\alpha$ crystallin molecules may be questioned. Indeed, the pair potential used by RMSA has been established for colloidal particles satisfying two conditions :

$$
k \sigma \ll 1 \text { and } e \psi_{0} \ll k_{\mathrm{B}} T \text {. }
$$

Neither condition is fulfilled by $\alpha$-crystallins since $k \sigma$ may be estimated a priori to vary from 8 at 17 mequiv to 23 at 150 mequiv while $e \psi_{0} / k_{\mathrm{B}} T$ can reach the value of 1 at 17 mequiv, if the number of charges is of the order of 60 . Fortunately the formulas describing screened coulombic interactions have been extended to less restrictive situations. For example, Bell et al. [12] calculated for $k \sigma \gg 1$ a generalized potential :

$$
\begin{aligned}
& v(r)=2 \pi \varepsilon_{0} \varepsilon \sigma\left(k_{\mathrm{B}} T / e\right)^{2}(2 r-\sigma / 2 r) \times \\
& \quad \times Y^{2} \ln [1+(\sigma / 2 r-\sigma) \exp (-k(r-\sigma))]
\end{aligned}
$$

with $Y=4 \tanh \left(e \psi_{0} / 4 k_{\mathrm{B}} T\right)$. It turns out that, for $\alpha$-crystallins, $Y$ deviates from $e \psi_{0} / k_{\mathrm{B}} T$ by less than $3 \%$. In addition

$$
\begin{aligned}
(2 r-\sigma / 2 r) \ln [1+(\sigma / 2 r-\sigma) \times \\
\times \exp (-k(r-\sigma))]
\end{aligned}
$$

reduces to

$$
(\sigma / 2 r) \exp (-k(r-\sigma)) \text { when } k(r-\sigma) \gg 1,
$$

a condition roughly equivalent to (7). Finally equation (8) reduces to equation (4) in the experimental situations where (7) is satisfied.

Thus, the model developed by Hayter and Penfold [3] and Hansen and Hayter [4] is pertinent for the description of $\alpha$-crystallin interactions except perhaps at low $I$ and high concentration, for which SAXS might probe an interaction potential quite different from that of equation (4). However, structure studies are not extremely sensitive to the precise shape of the potential.

\section{Results.}

3.1 FORM FACTOR. - The renormalized experimental curves recorded at 3 concentrations and two ionic strengths are shown in figure 1 . It can be seen that the concentration effect is limited to the low angle part of the scattering curves, which become identical
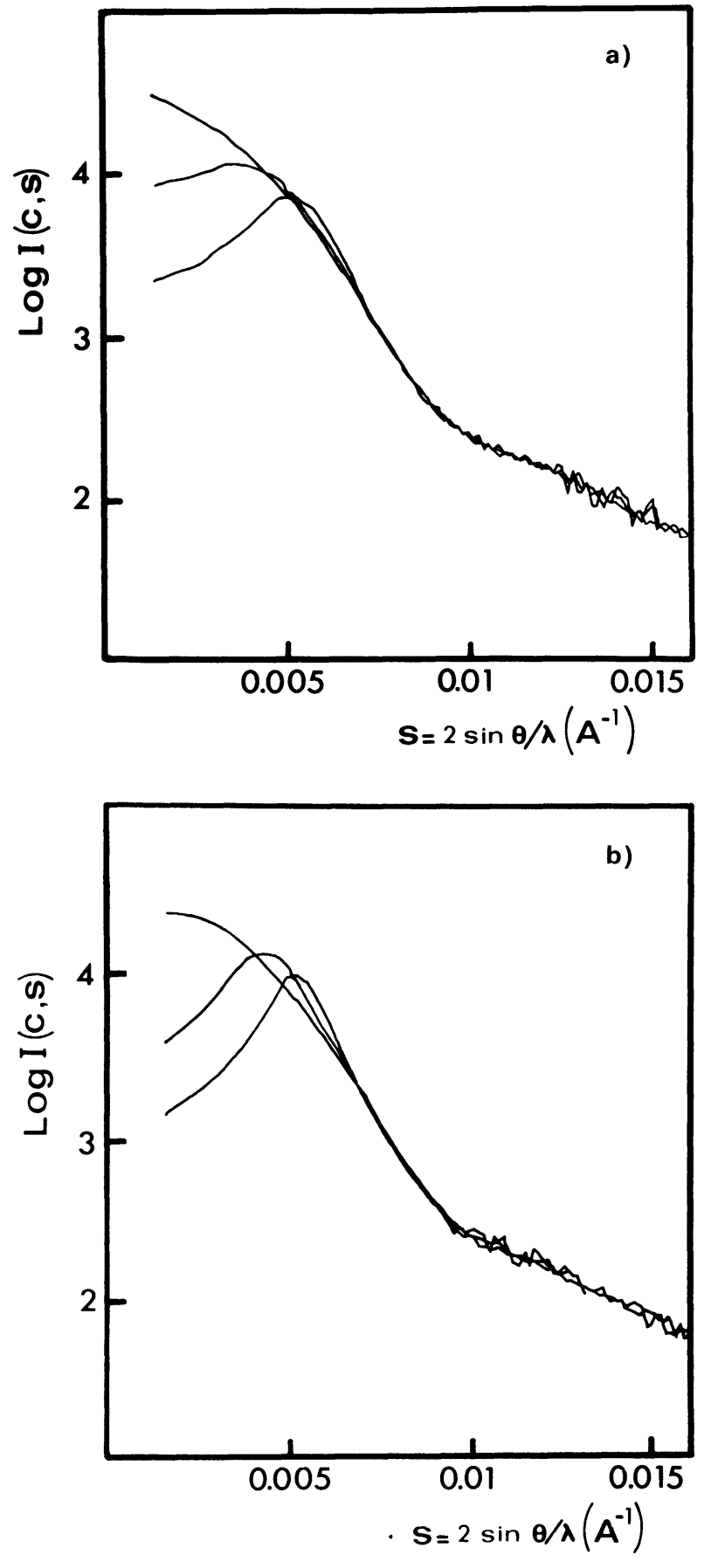

Fig. 1. - X-ray scattering curves recorded at $I=150$ mequiv (a) and $I=17$ mequiv (b). At $I=$ 150 mequiv, concentrations were $0.01 \mathrm{~g} / \mathrm{cm}^{3}, 0.095 \mathrm{~g} / \mathrm{cm}^{3}$ and $0.205 \mathrm{~g} / \mathrm{cm}^{3}$. At $I=17$ mequiv, concentrations were $0.010 \mathrm{~g} / \mathrm{cm}^{3}, 0.107 \mathrm{~g} / \mathrm{cm}^{3}$ and $0.184 \mathrm{~g} / \mathrm{cm}^{3}$. Data are subtracted from background and renormalized but otherwise uncorrected.

for $s$ values well below those where the asymptotic trend is reached. From the identity of the scattering curves at medium and high angles it can be concluded that the form factor remains essentially the same whatever the concentration, as expected for proteins.

Average $M$ and $R_{\mathrm{g}}$ deduced from Guinier plots may vary slightly according to the $\alpha$-crystallin frac- 
tion selected ( $M$ within $\pm 15 \%$ and $R_{\mathrm{g}}$ within $\pm 5 \%$ ). In the present case, a $M$ of $1.1 \times 10^{6}$ (i.e. 55 subunits) associated with an $R_{\mathrm{g}}$ of $67 \AA$ was obtained. Beyond the central peak, the scattering curve displays one broad shoulder around $s=$ $0.01 \AA^{-1}$, but no well defined minimum (Fig. 1), which indicates significant departure from sphericity in this region. All these features were accounted for by a three layer model, displaying tetrahedral symmetry, in which the polydispersity corresponds to variable filling of the external layers [2] (Fig. 2). Because of the slight variability from one preparation to another, and because the form factor of such a system cannot be modelled by any simple analytical expression (except in the Guinier region), an experimental low concentration curve recorded in exactly the same conditions (yet with a long accumulation time) was used as the form factor. The $c=$ $0.01 \mathrm{~g} / \mathrm{cm}^{3} I=150$ mequiv curve was chosen as a compromise between the contradictory requirements of sufficient statistics yet negligible interactions in the recorded range.

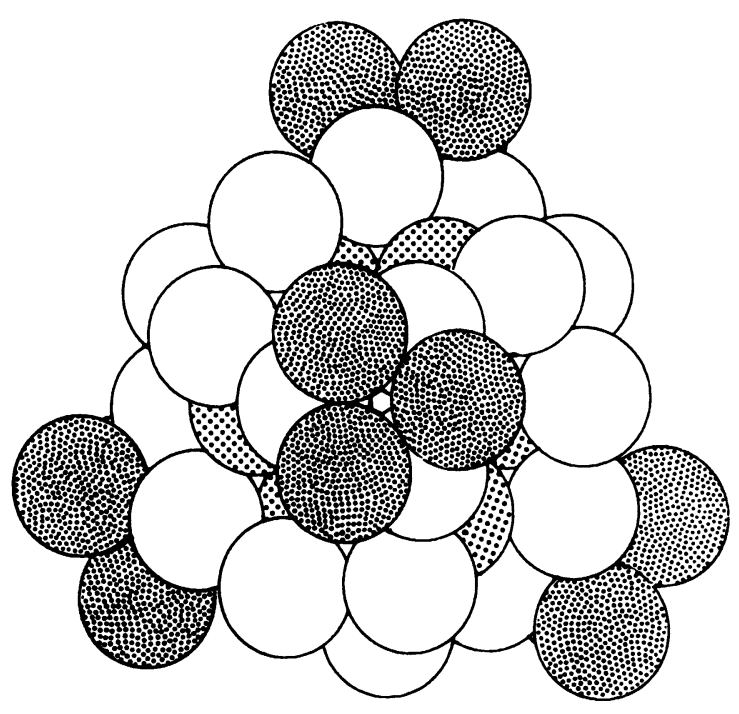

Fig. 2. - Schematic representation of the model developed in reference [2] for the quaternary structure of $\alpha$ crystallin. Subunits have been modelled by spheres. This picture corresponds to a 48 subunits particle (12 on the first layer, 24 on the second one and 12 on the third one). The model is seen from a 3 -fold axis.

3.2 STRUCTURE FACTORS. - The experimental structure factors determined by equation (2) at $I=150$ mequiv and $I=17$ mequiv and for two concentrations are shown in figure 3 .

These experimental structure factors start from values lower than 1 near $s=0$, rise to a maximum around $s_{\mathrm{m}}=5.5 \times 10^{-3} \AA^{-1}$ (i.e. $q_{\mathrm{m}}=3.5 \times$ $10^{6} \mathrm{~cm}^{-1}$ ) (height of the peak higher than 1) and then fall to 1 . When $c$ increases, the value of $S_{\text {exp }}$ near the origin decreases and the height of the peak increases. When $I$ decreases at a given $c$, the height of the peak increases while the peak position is shifted towards lower $s$ values. All these features are in qualitative agreement with the predictions of the model developed in references [3-4]. Beyond the maximum, however, no secondary minimum or maximum are observed, which may reflect polydispersity and/or departure from sphericity.

The model involves three parameters, the volume fraction $\phi$ occupied by the particles, the diameter $\sigma$ and the charge $Z$. With hard core particles, $\phi$ and $\sigma$ are coupled. As the molecular weight is known and invariant with concentration, $\phi$ and $\sigma$ are related by :

$$
\phi=\frac{c \mathcal{N}}{M} \frac{\pi \sigma^{3}}{6} .
$$

This relation left us with only two independent adjustable parameters $\phi$ and $Z$. A first series of calculations showed that, at $I=150$ mequiv, the fit was, as could be expected, relatively insensitive to the value of $Z$. Interestingly, to fit satisfactorily the $S_{\text {exp }}(c, s)$ values at low $s$, volume fractions $\phi$ larger than $1.6 \times c$, i.e. much higher than the dry volume fractions which correspond to $\phi=0.74 \times c$, were needed. Such a result is not surprising given that $\alpha$ crystallin quaternary structure is far from compact, but means that the excluded volume fraction cannot be guessed and must be experimentally determined.

At $I=17$ mequiv $Z$ becomes a sensitive parameter. If both $\phi$ and $Z$ were left free to vary, a wide range of coupled $(\phi, Z)$ values were found to satisfactorily fit the low angle part of the structure factor (typically, from $\phi / c=3, Z=1$ up to $\phi / c=1, Z=80$ ).

This first step of the analysis led us to formulate additional hypotheses. We further assumed that neither the $\phi / c$ ratio nor the number of effective charges did vary with $I$. Such assumptions are critically examined in the next section.

The following strategy was then adopted to fit the scattering curves : in a first run, we attempted to fit the $I=150$ mequiv data with $\phi$ as the single variable and $Z=1$ (step $I$ in Tab. I). The relation thus obtained between $\phi$ and $c$ was assumed to be valid also at $I=17$ mequiv, for which the fit was done on the basis of charge alone (step II). The charge thus determined was reinjected at $I=150$ mequiv (step III) and the process reiterated until convergence. As the effect of polydispersity seemed to be all the more pronounced with increasing $s$, we looked for a fit at low $s$ values $\left(0.0013 \AA^{-1}<\right.$ $s<0.0040 \AA^{-1}$ ) where the polydispersity was neglected. The best fits obtained that way are shown in figure 3 and the best fit parameters are given in table I. Note that convergence was obtained in one iteration.

It can be seen that the agreement is excellent in 
Table I. - Parameters giving the best fit between the model developed in references (3) and (4) and the experimental structure factors shown in figure 3. Procedures used in stemps I to IV are described in the text. The underlined values are those which are held fixed in each step.

\begin{tabular}{|c|c|c|c|c|c|c|}
\hline $\begin{array}{c}\text { Step } \\
\mathrm{n}^{\mathrm{o}}\end{array}$ & $\begin{array}{c}I \\
\text { (mequiv) }\end{array}$ & $\begin{array}{c}C \\
\left(\mathrm{~g} / \mathrm{cm}^{3}\right)\end{array}$ & $\phi$ & $\phi / c$ & $Z$ & $\sigma(\AA)$ \\
\hline I & 150 & 0.095 & 0.174 & 1.83 & 1 & 185.6 \\
150 & 0.205 & 0.347 & 1.69 & $\underline{1}$ & 180.8 \\
\hline II & 17 & 0.107 & 0.188 & 1.76 & 50 & 183.1 \\
\hline III & 17 & 0.184 & $\underline{0.324}$ & 1.76 & 47 & $\frac{183.1}{-}$ \\
\hline IV & 150 & 0.095 & 0.167 & 1.76 & 49 & 183.1 \\
& 17 & 0.107 & 0.182 & 1.70 & 52 & 181.1 \\
\hline
\end{tabular}

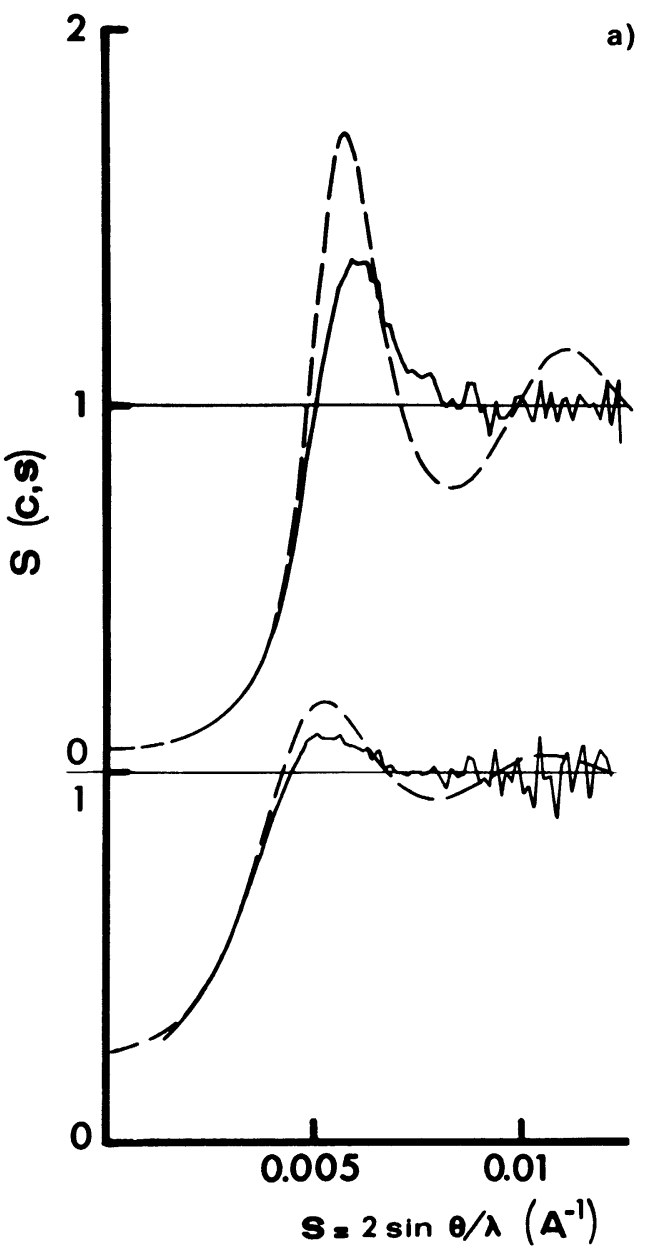

the $s$ domain used to fit the parameters and reasonably good at higher $s$ values. Furthermore, the calculated peak position is in agreement with the observed one, even though the particle diameter was calculated from equation (9) and was not independently adjusted. At $I=150$ mequiv, the $\phi / c$ values found were close to each other at both concentrations. The average $\phi / c$ value, when used at $I=17$ mequiv, resulted in the same number of charges for both concentrations. These results indicate the adequacy of the model to describe experimental structure factors of $\alpha$-crystallin dispersions.

\section{Discussion.}

Several hypotheses were used for the data analysis. The first one was that the experimental curve obtained at $c=0.01 \mathrm{~g} / \mathrm{cm}^{3}, I=150$ mequiv could be taken as the form factor. The invariance of the particle size and shape with concentration is confirmed by the constancy of the best fit parameters obtained. Moreover, using the parameters determi- a)

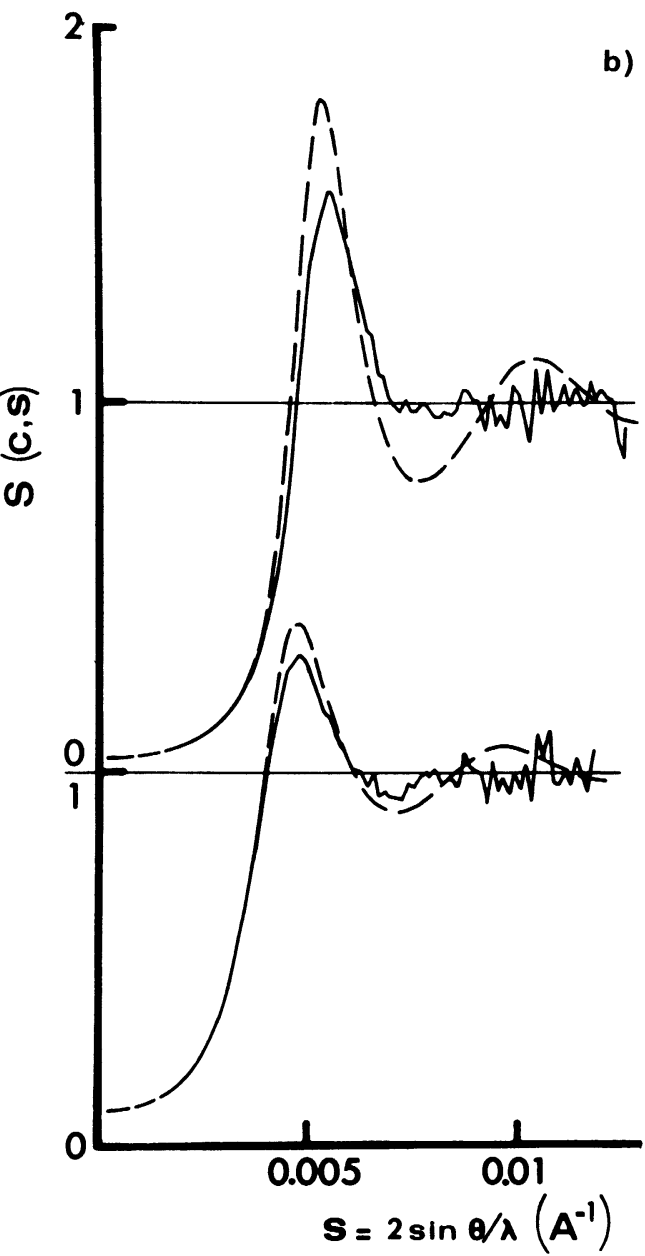

Fig. 3. - Experimental (full lines :- - and theoretical (dotted lines : ----) structure factors obtained as explained in the text. (a) $: I=150$ mequiv ; $c=0.095 \mathrm{~g} / \mathrm{cm}^{3}:$ lower curve ; $c=0.205 \mathrm{~g} / \mathrm{cm}^{3}:$ upper curve. (b) $: I=17$ mequiv ; $c=0.107 \mathrm{~g} / \mathrm{cm}^{3}$ : lower curve ; $c=0.184 \mathrm{~g} / \mathrm{cm}^{3}$ : upper curve. 
ned at high concentration to calculate the structure factor at $c=0.01 \mathrm{~g} / \mathrm{cm}^{3}$ and $I=150$ mequiv, show that $S_{\text {th }}$ is already equal to 0.96 at $s=1 / 2 \pi R_{\mathrm{g}}$ and is equal to 1 within $2 \%$ for $s>1.3 / 2 \pi R_{\mathrm{g}}$. That may explain why interaction effects were not detected in the $c=0.01 \mathrm{~g} / \mathrm{cm}^{3}, I=150$ mequiv scattering curve and legitimates the choice of this curve as the form factor.

To fit our experimental data with the model developed in references [3, 4], we further assumed that the effect of polydispersity could be neglected at low $s$ values. As can be seen in figure 3, the maximum of the experimental structure factor is lower by about $20 \%$ than the calculated one, and other minima or maxima are absent. According to Frenkel et al. [13], such a decrease in the maximum of the structure factor and the disappearance of subsequent oscillations arise from a size polydispersity of the order of 10 to $15 \%$. This is in agreement with our biochemical estimation of the size polydispersity of the $\alpha$-crystallin population used in this study. In previous experiments, where a narrower $\alpha$ crystallin distribution was used [14], the deviation of the experimental structure factor from the calculated one was smaller. A posteriori, the simulations performed in reference [13] indicate that a 10 to $15 \%$ polydispersity increases the $S(c, 0)$ values by at most $5 \%$ as compared to the monodisperse case. Neglecting the polydispersity for $s<0.004 \AA^{-1}$ thus does not impair the best fit parameters by more than a few \%. In contrast, the treatments of polydispersity which decouple sizes and positions [15] predict major deviations at low $s$ and minor deviations around the peak and could not account for our experimental data.

From figure 3 it even seems that the effect of polydispersity is less pronounced at $I=17$ mequiv than at $I=150$ mequiv. The charge polydispersity thus does not seem to be so critical. In this context, it is worth recalling that the maximum of $S(c, s)$ is close to the $s$ region where the form factor starts to depart from sphericity. Part of the difference between experimental and theoretical curves, particularly the absence of oscillations, might thus partly originate from deviations from sphericity.

The adequacy of the model to describe the experimental curves raises an additional question. Using the VO potential to describe the interactions between $\alpha$-crystallins implicitly assumes that Van der Waals attraction can be neglected. Verification of this assumption requires an evaluation of the Hamaker constant $A_{\alpha w}$, which determines the strength of the Van der Waals attraction between two $\alpha$-crystallin proteins immersed in an aqueous solvent. Following reference [16], one can approximate $A_{\alpha \mathrm{w}}$ by

$$
A_{\alpha \mathrm{w}}=\left(\sqrt{A_{\alpha \alpha}}-\sqrt{A_{\mathrm{ww}}}\right)^{2}
$$

where $A_{\alpha \alpha}$ and $A_{\text {ww }}$ stand for the Hamaker constant between respectively two $\alpha$-crystallin and two water molecules in vacuum. $A_{\mathrm{ww}} \simeq 11 k_{\mathrm{B}} T$ [16] and the Hamaker constant for monomeric globular proteins (such as BSA) in vacuum was evaluated to be $2.5<A_{\mathrm{pp}}<6 k_{\mathrm{B}} T$ [17]. Taking $A_{\alpha \alpha}=A_{\mathrm{pp}}$ would lead to $0.75<A_{\mathrm{pw}}<3 k_{\mathrm{B}} T$. However, because the excluded volume of $\alpha$-crystallins is much larger than the dry volume and contains a lot of water, $A_{\alpha \mathrm{w}}$ is severely reduced as compared to $A_{\mathrm{pw}}$. Following [16], one can evaluate $A_{\alpha \alpha}$ as :

$$
A_{\alpha \alpha}=x A_{\mathrm{ww}}+(1-x) A_{\mathrm{pp}}
$$

where $x$ is the volume fraction of water inside the excluded volume. With $\phi / c=1.7$ and $x=0.57$ one ends up with a Hamaker constant of $0.11<A_{\alpha \mathrm{w}}<$ $0.36 k_{\mathrm{B}} T$ i.e. 8 to 12 times smaller than $A_{\mathrm{pw}}$ and $\sim 40$ times smaller than the Hamaker constant describing the attraction between micelles [18].

Using this value for the Van der Waals attraction and a charge $Z=50$ for the Coulombic repulsion, one finds that, at $I=17$ mequiv, the total interparticle potential would already be of the order of $3 k_{\mathrm{B}} T$ at $3 \AA$ from the surface. It is thus likely that the attractive part of the potential is negligible. The situation is less clear at $I=150$ mequiv, where the attractive term would only be counterbalanced by the repulsive term $5 \AA$ away from the particle surface. However, as the Van der Waals attraction need not enter into consideration for the fit, other forces, due for instance to solvent structuration, might also play a role at short distances.

Since $\phi / c$ and $Z$ could not both be determined from a single experiment, our fitting procedure also assumed that $\phi / c$ and $Z$ did not depend upon $I$. The validity of such an assumption is difficult to check. If $\phi / c$ actually varies as a function of $I$, this would alter our estimation of the charge. However, as can be seen from table $I$, only physically unrealistic changes in $\phi / c$ would change the order of magnitude of $Z$. The effective charge $Z$, which determines the surface potential, might also be slightly different at $I=17$ mequiv and $I=150$ mequiv if the charge « association " [19] is affected by the amount of salt in the buffer. Such a difference could not be detected in our experiments, since the $\phi / c$ determination at $I=150$ mequiv is almost insensitive to the charge. The constancy of the best fit parameters obtained at two concentrations, and the coherence of our results with other data from the literature (next section) seem however to indicate that the assumption was justified.

In the present analysis, we also neglected possible $\phi / c$ variations as a function of $c$ by taking $\phi / c$ values averaged over the two studied concentrations. Work presently under way using wider $s$ and $c$ ranges seem to indicate a slight decrease with $c$ of the $\phi / c$ ratio. 
The best fit parameter values obtained can be compared with corresponding values found in the literature. The hard sphere diameter $\sigma=181 \AA$ calculated from $\phi / c$ is close to the hydrodynamic diameter $2 R_{\mathrm{h}}=190 \AA$ [5] measured by photon correlation spectroscopy. It lies in between the diameter of the sphere which would have the same radius of gyration as $\alpha$-crystallins, $174 \AA$, and the outer diameter of the particle that was inferred from the quaternary structure modelling of $\alpha$-crystallins to be about $200 \AA$ [2]. These comparisons indicate that the effective hard sphere diameter in such a system is indeed close to the outer envelope of the rotating particle. In this respect, it is worth stressing that the excluded volume per particle is entirely different from the so-called hydrated volume which is calculated, in scattering experiments, from the Patterson function at the origin. In the present study, the hydrated volume was found to be of the order of 1.2 to 1.3 times the dry volume (unpublished results), while the excluded volume is 2.3 times the dry volume. This important distinction between hydrated volume and excluded volume was omitted by Chen in reference [20]. If both values may be expected to be close to each other when the protein under study has a compact quaternary structure, the $\alpha$-crystallin example demonstrates that such a situation is far from being a general one.

The effective charge $Z=50$ obtained from the low $I$ data correctly compares with the evaluation $Z=46$ deduced from electrophoretic measurements [21]. This result is encouraging since electrophoretic measurements probe a $\zeta$-potential which might not be too far from the surface potential $\psi_{0}$. In contrast, the intrinsic charge $Z=85$ deduced from the subunit composition [22] is higher. Such discrepancies are usually ascribed to partial « condensation " [23] or « association » [19] of the counter-ions on the particle surface.

From the whole of these results, the Renormalized Mean Spherical Approximation using a VO potential appears well suited to describe $\alpha$-crystallin dispersions. This model should also be valid for other protein dispersions where the repulsive interactions are expected to be predominant. It seems reasonable, however to predict that, as is often the case with biological systems, there will be a variety of behaviours depending upon the particular protein species under study and the physico-chemical conditions. The balance between attractive and repulsive interactions can be modified within a range of $\mathrm{pH}$ (i.e. of charges) or $I$ (i.e. of screening) that does not affect the quaternary structure of the particle. This interesting property is not generally fulfilled by colloids such as micelles or microemulsions. In addition, by using different protein species, parameters such as compactness and shape of the colloidal particle can be varied. These parameters in turn modulate both the strength of the Van der Waals interaction and the ratio between excluded volume and concentration. To prevent coagulation, increasing the amount of water contained in the protein excluded volume could thus be equivalent to coating silica beads with a polymeric layer. $\alpha$-crystallin quaternary structure might thus reflect the need to reduce Van der Waals attractions, hence aggregate formation, to maintain transparency in the lens cytoplasm.

Proteins thus provide a natural source of model systems to study colloidal dispersions. The protein diversity, however, calls for caution in handling data analysis. In particular, variation of the physicochemical conditions may, in some cases, be essential to determine parameters such as the excluded volume fraction and the effective charge with sufficient precision. Any attempt to correct a priori for concentration effects as suggested for instance by Chen [20] would often lead to erroneous results.

\section{Conclusion.}

We have shown that interparticle interactions in $\alpha$ crystallin dispersions can be correctly described by screened coulombic potentials, both at $I=150$ and 17 mequiv and over a wide concentration range. Values deduced from this study for the hard sphere diameter $\sigma$, the effective charge $Z$ and the $\phi / c$ ratio are both self consistent and comparable to independent evaluations.

This type of structural analysis shows that, although rarely considered, protein-protein interactions do play a major role in the spatial organization of dense cellular media. In the case of the eye lens, alteration of the protein-protein interactions due to chemical modifications might be one of a number of factors that may lead to loss of transparency [1], i.e. to cataract.

Finally, this study shows the adequacy of using $\alpha$ crystallins as model systems for colloids. These proteins offer the possibility of testing the effect of charge and of polydispersity on the structure factor. The choice of other proteins should allow us to vary other parameters, such as size, shape, compactness or the balance between attractive and repulsive interactions. At present, the understanding of their interactions make $\alpha$-crystallin proteins a good model system for studying the dynamics of concentrated colloidal dispersions. The results obtained using photon correlation spectroscopy are described in part II [5].

\section{Acknowledgments.}

We thank Linda Sperling for critical reading of the manuscript, Patrice Vachette for help during the Xray experiments and Thomas Zemb for advice concerning the computer program. We also thank 
Jean-Claude Dedieu for drawing the figures and Brigitte Krop for expert technical assistance. This work was supported in part by an MRT grant (Interface Physique Biologie) and an INSERM grant (contrat de recherche externe subsidized by the CNAMTS) and was pursued within the scope of the European Community Concerted Action on Cellular Aging and Disease (EURAGE).

\section{References}

[1] Delaye, M., Tardieu, A., Nature 302 (1983) 415.

[2] Tardieu, A., Laporte, D., Licinio, P., Krop, B., Delaye, M., J. Mol. Biol. 192 (1986) 711.

[3] Hayter, J. B., Penfold, J., Mol. Phys. 42 (1981) 109.

[4] Hansen, J. P., Hayter, J. B., Mol. Phys. 46 (1982) 651.

[5] Licinio, P., Delaye, M., Livesey, A. K. and Leger, L., J. Physique 48 (1987).

[6] Bordas, J., Koch, M. H. J., Clout, P. N., DorRington, E., Boulin, C., GAbriel, J., J. Phys. $E(1980) 938$.

[7] Luzzati, V., Tardieu, A., Ann. Rev. Biophys. Bioeng. 9 (1980) 1.

[8] Luzzati, V., Witz, J., Nicolaieff, A., J. Mol. Biol. 3 (1961) 367.

[9] Belloni, L., Chem. Phys. 99 (1985) 43 and J. Chem. Phys. 85 (1986) 519.

[10] Nagele, G., Klein, R., Medina-Noyola, N., $J$. Chem. Phys. 83 (1985) 2560.

[11] Verwey, E. J. W., OVERBEeK, J. T. G. in Theory of the stability of lyophobic colloids (Elsevier) 1948.
[12] Bell, G. M., Levine, S., Mc Cartney, L. N., $J$. Coll. Interface Sci. 33 (1970) 335.

[13] Frenkel, D., Vos, R. J., De Kruif, C. G., VRiJ, A., J. Chem. Phys. 84 (1986) 4625.

[14] Tardieu, A., Delaye, M. in The lens : transparency and cataract (1986) G. Duncan Ed. Topics in aging research in Europe vol. 6, p. 49.

[15] Kotlarchyк, M., Chen, S. H., J. Chem. Phys. 79 (1983) 2461.

[16] Visser, J., Advan. Colloid Interface Sci. 3 (1972) 331.

[17] Srivastava, S. N., Z. Phys. Chem. 233 (1966) 237.

[18] Corti, M., Degiongio, V., J. Phys. Chem. 85 (1981) 711.

[19] Bjerrum, N. K., Danske Vidensk. Selsk. 7 n 9 (1926).

[20] Chen, S.-H., Bendedouch, D., Meth. Enzymol. 130 (1986) 79.

[21] Siezen, R. J., Owen, E. A., Biophys. Chem. 18 (1983) 181.

[22] Siezen, R. J., Bindels, J. G., Hoenders, H. J., Eur. J. Biochem. 91 (1978) 387.

[23] Manning, G. S., J. Chem. Phys. 51 (1969) 924. 